\title{
Secular Trend and Risk Factors for Antimicrobial Resistance in Escherichia coli Isolates in Switzerland 1997-2007
}

\author{
L. Blaettler, D. Mertz, R. Frei, L. Elzi, A.F. Widmer, M. Battegay, U. Flückiger
}

\begin{abstract}
Background: Antibacterial resistance in Escherichia coli isolates of urinary infections, mainly to fluoroquinolones, is emerging. The aim of our study was to identify the secular trend of resistant $E$. coli isolates and to characterize the population at risk for colonization or infections with these organisms.

Patients and Methods: Retrospective analysis of 3,430 E.coli first isolates of urine specimens from patients admitted to the University Hospital Basel in 1997, 2000, 2003, and 2007.

Results: Resistance to ciprofloxacin, trimethoprim/sulfamethoxazole, and amoxicillin/clavulanate has increased over the 10 -year study period (from $1.8 \%$ to $15.9 \%, 17.4 \%$ to $21.3 \%$, and $9.5 \%$ to $14.5 \%$, respectively). A detailed analysis of the 2007 data revealed that independent risk factors for ciprofloxacin resistance were age $(5.3 \%<35$ years of age to $21.9 \%$ in patients $>75$ years; odds ratio [OR] 1.29 per 10 years, $95 \%$ confidence interval [CI] 1.15-1.45, $\mathrm{p}<0.001$ ) and male gender (OR 1.59, 95\% CI 1.05-2.41, $\mathrm{p}=0.04$ ). In contrast, nosocomial $E$. coli isolates were associated with lower odds of ciprofloxacin resistance (OR $0.51,95 \%$ CI $0.28-0.67, p<0.001)$. The frequency of resistant isolate rates was not influenced by the clinical significance (i.e., colonization vs urinary tract infection, UTI) or by whether the urine was taken from a urinary catheter. Importantly, the increase in ciprofloxacin resistance paralleled the increase in ciprofloxacin consumption in Switzerland (Pearson's correlation test $\left.R^{2}=0.998, p=0.002\right)$. Of note, resistance was less frequent in isolates sent in by general practitioners. However, after adjustment for age and gender, only resistance against amoxicillin/clavulanate was found to be less frequent (0R $0.34,95 \%$ CI $0.16-0.92, \mathrm{p}=0.03$ ).

Conclusion: Our study reveals that resistance rates have been increasing during the last decade. Published resistance rates may lack information due to important differences regarding age, gender, and probable origin of the isolates. Empirical therapy for UTI should be guided more on individual risk profile and local resistance data than on resistance data banks.
\end{abstract}

Infection 2009; 37: 534-539

DOI 10.1007/s15010-009-8457-0

\section{Introduction}

Urinary tract infections (UTIs) are a common cause of morbidity in otherwise healthy persons and mostly affect women [1]. Uncomplicated UTIs are treated empirically without culturing the urine [2]. Consequently, adequate knowledge of the regional resistance pattern of uropathogens is essential.

Escherichia coli (E. coli) is the primary UTI pathogen and responsible for $75-90 \%$ of cases in the community [3, 4]. Most of the relevant studies carried out to date refer to hospitalized patients, and the susceptibility of E. coli varies considerably in different parts of the world [5-9]. Trimethoprim/sulfamethoxazole (TMP/SMX) is the empiric treatment of choice for UTIs, if the local resistance rate is less than $10-20 \%$ [10]. However, various studies have demonstrated that fluoroquinolones are increasingly used $[9,11]$, probably because of concerns about increasing resistance to TMP/SMX [12]. As a result of this increased fluoroquinolone use, resistance to this antibiotic class also is increasing among uropathogens $[8,13,14]$.

Data on host factors for colonization or infection with a resistant E. coli strain are not conclusive. Various studies have shown that $E$. coli resistance may be predicted by age $[4,15,16]$, male sex $[4,15]$, nosocomial infection [15], traveling habits [3,17], ethnicity [3,18], known malignancies [19], and previous antibiotic treatment $[16,20]$.

As the clinical outcome is influenced by the adequacy of the empiric antibiotic treatment [21, 22], knowledge about the supposed probability of a resistant $E$. coli strain in a individual patient is crucial. As such, current guidelines based on overall cumulative antimicrobial suscepti-

\footnotetext{
L. Blaettler, D. Mertz, L. Elzi, M. Battegay, A.F. Widmer,

U. Flückiger (corresponding author)

Division of Infectious Diseases and Hospital Epidemiology, University

Hospital Basel, Petersgraben 4, 4031 Basel, Switzerland;

Phone: $(+41 / 61)$ 265-2525, Fax: -3198 ,

e-mail: flueckigerU@uhbs.ch

R. Frei

Microbiology Laboratory, University Hospital Basel, Basel, Switzerland

L. Blaettler and D. Mertz contributed equally to this work.
}

Received: December 1, 2008; revision accepted: April 8, 2009;

Published online: November 21, 2009 
bility test data may be intrinsically limited. Given this background, the aim of our study was to analyze the limitations of data on cumulative resistance rates by characterizing the population at risk for colonization or infections with $E$. coli isolates resistant to the most frequently prescribed antimicrobial compounds. We also analyzed the secular trend of resistance in $E$. coli isolates in northwestern Switzerland.

\section{Materials and Methods Setting}

The University Hospital Basel is a tertiary care center with 780 beds serving approximately 27,000 admissions annually. All E. coli isolates grown from urine specimens in our microbiological laboratory in 1997, 2000, 2003, and 2007 were included in our study as being representative of the trend over the last 10 years. The patients were either in- or out-patients of the University Hospital Basel. Additionally, in 2007, isolates from urine specimens sent by general practitioners $(\mathrm{n}=106)$ were analyzed.

For susceptibility testing and the yearly cumulative antimicrobial susceptibility test data, the microbiology laboratory followed the yearly updated Clinical and Laboratory Standards Institute (CLSI) guidelines [23]. Intermediate and resistant isolates were combined into one interpretative category (resistant). The study was approved by the Ethics Committee of the Cantons Basel-Stadt and Basel-Land (EKBB).

\section{Secular Trends in Antimicrobial Resistance}

The susceptibility patterns of all E. coli first isolates of urine specimens from patients treated in 1997, 2000, 2003, and 2007 against the antimicrobial compounds most frequently used in the setting of UTIs were analyzed: amoxicillin/clavulanate, TMP/ SMX, ciprofloxacin, and nitrofurantoin. The IMS HEALTH (Hergiswil, Switzerland), an international supplier of informa- tion and services for the pharmaceutical and health industry, provided data on ciprofloxacin, amoxicillin/clavulanate, and TMP/SMX use in Switzerland in terms of the number of packages sold annually.

\section{Characteristics of Patients with E. coli Urinary Isolates in 2007}

We compiled data on the clinical background and the origin of the 2007 isolates. Based on patient files, we distinguished asymptomatic bacteriuria or colonization from symptomatic UTI or urosepsis. A UTI was diagnosed if an infection was mentioned in the charts and/or the patient was treated accordingly. Urosepsis was defined if a UTI was diagnosed in combination with the diagnosis of sepsis according to the physician(s) in charge. In addition, urine isolates from spontaneous micturition or singlecatheterism were compared to isolates from urinary catheters. If the culture was taken more than $48 \mathrm{~h}$ after admission, the isolate was defined as being nosocomially acquired.

\section{Statistical Analysis}

For the univariate analysis, the $\chi^{2}$-test for binary data and the Mann-Whitney U-test for continuous data were used. Multivariate analysis was performed using logistic regression. The results are reported using odds ratios (OR) and $95 \%$ confidence intervals $(95 \% \mathrm{CI})$. To evaluate the trend of emergence of resistance over time, the $\chi^{2}$-test for trend was used. To correlate the emergence of resistance and the increase in antibiotic use, we used Pearson's correlation test.

\section{Results}

Secular Trends in Antimicrobial Resistance In the years of interest, i.e., 1997, 2000,2003, and 2007, a total of 3,430 first isolates of $E$. coli from urine cultures were recorded. The median age of the patients was $65,64,63$, and 72 years, respectively, and the percentage of females was
Figure 1. Development of resistance and ciprofloxacin use over a 10-year period in 3,430 urinary first isolates of $E$. coli. Asterisk: $\mathrm{p}$-values using the $\chi^{2}$-test for trend.

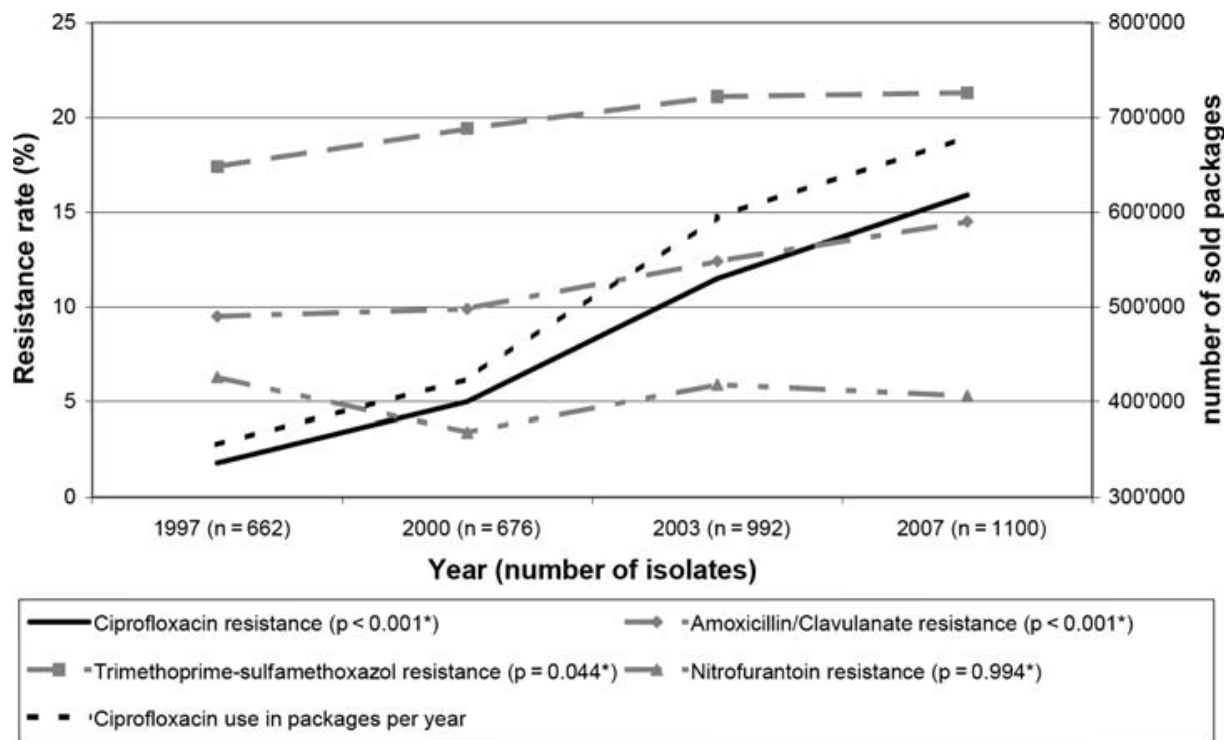

* P-values using Chi-Square test for trend 


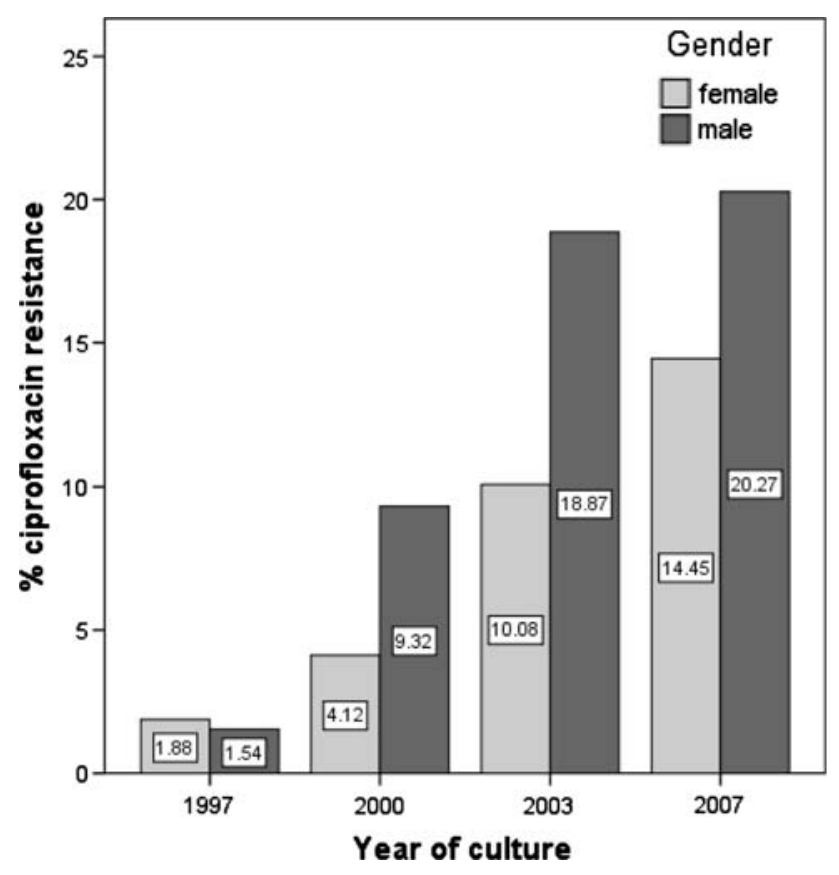

Figure 2. Differences in development of ciprofloxacin resistance in urinary first isolates of $E$. coli in male and female patients.

$80.4 \%, 82.5 \%, 84.0 \%$, and $79.5 \%$, respectively. There was a statistically significant increase in resistance over time for all of the antibiotics investigated with the exception of nitrofurantoin (Figure 1). Notably, a major increase was observed for ciprofloxacin (overall from $1.8 \%$ to $15.9 \%$ ), which cor- relates significantly with the increase in ciprofloxacin usage in Switzerland (Pearson's correlation test $\mathrm{R}^{2}=0.998$, $\mathrm{p}=0.002$ ). A similar trend was observed for amoxicillin/ clavulanate $\left.\mathrm{R}^{2}=0.926, \mathrm{p}=0.074\right)$. In contrast, there was an inverse correlation for TMP/SMX resistance and use of TMP/SMX $\left.\mathrm{R}^{2}=-0.989, \mathrm{p}=0.011\right)$ : Despite a steady reduction in the number of packages sold annually since 1990, we found that resistance rates had increased since 1997.

It is notable that ciprofloxacin-resistant isolates were slightly more common in females in 1997 (Figure 2) but that over time, the increase in ciprofloxacin resistance was more prominent in males. A linear increase in the number of E. coli isolates resistant to ciprofloxacin was observed with increasing age from the year 2003 onwards, reaching a prevalence of $5.3 \%$ in patients younger than 35 years to $21.9 \%$ in those older than 75 years $(\mathrm{p}<0.001)$ in 2007 (Figure 3). In patients older than 75 years, the resistance against ciprofloxacin was even more frequent than that against TMP/SMX (Figure 3). For amoxicillin/clavulanate, the resistance of $E$. coli varied in the different age groups, with a trend to a higher resistance rate in older patients. Nitrofurantoin resistance was found to be below $10 \%$ in patients of all age groups. Among the 335 ciprofloxacin-resistant strains analyzed in this study, 171 (51\%) were co-resistant against TMP/SMX.

\section{Characteristics of Patients Treated in 2007 with E. coli Isolates in Urine Specimens}

1,100 patients treated in 2007 for whom $E$. coli was identified in urinary first samples were further investigated. The patient characteristics are given in table 1 . Detailed clinical information was available in 893 cases
Figure 3. Age dependence of resistance rates in E. coli first isolates in urine specimens of patients treated in 2007.

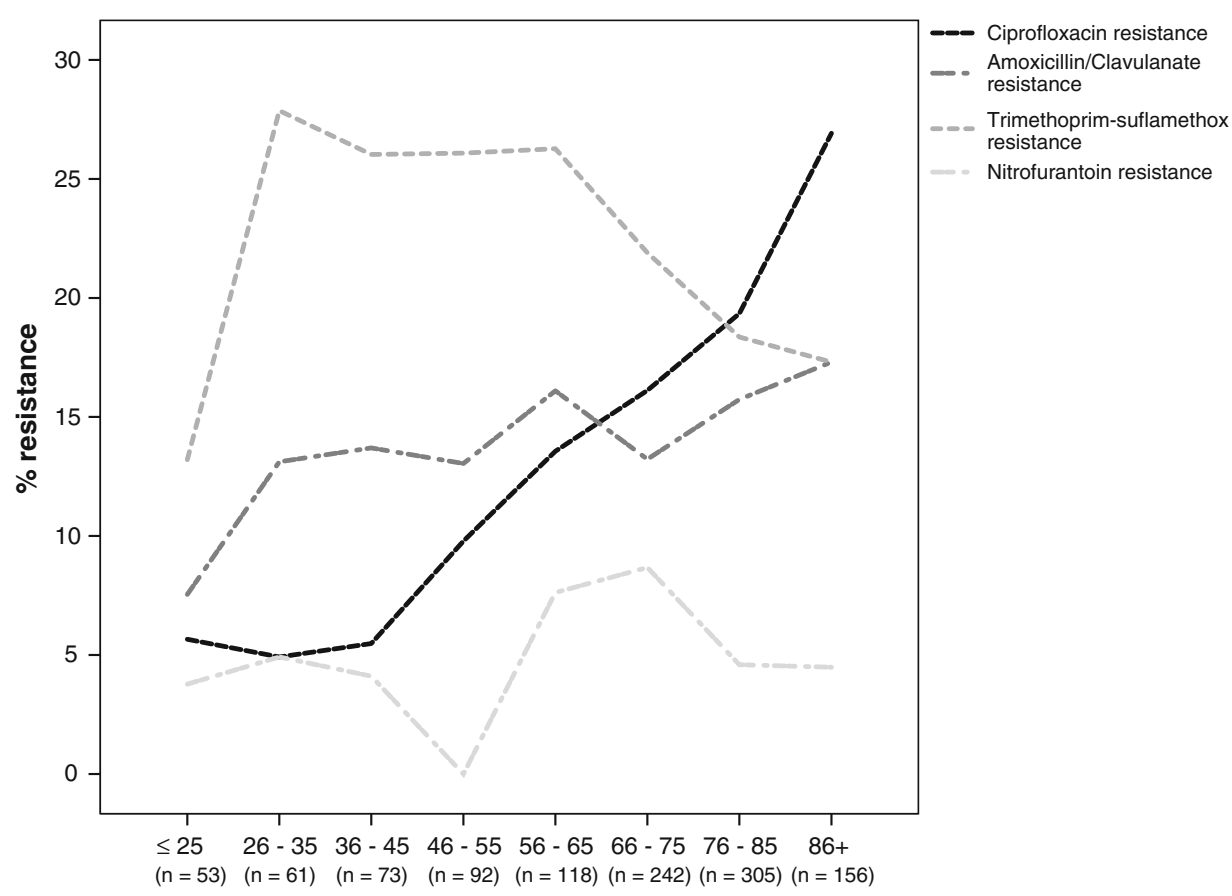

Patient age in years ( $n=$ number of isolates) 


\begin{tabular}{|c|c|}
\hline Characteristics & Values \\
\hline Female gender, n (\%) & $874(79.5)$ \\
\hline Age, median (IQR) & $72(55-82)$ \\
\hline \multicolumn{2}{|l|}{ Origin of isolate, $\mathrm{n}(\%)$} \\
\hline $\begin{array}{l}\text { Spontaneous micturition or one-time } \\
\text { catheterization }\end{array}$ & $912(82.9)$ \\
\hline Urinary catheter & $188(17.1)$ \\
\hline Nosocomial isolate & $323(29.4)$ \\
\hline Diagnosis available, $\mathrm{n}(\%)$ & $893(81.2)$ \\
\hline Asymptomatic bacteriuria & $269(30.1)$ \\
\hline Urinary tract infection & $545(61.0)$ \\
\hline Urosepsis & $70(7.8)$ \\
\hline Other infections ${ }^{a}$ & $9(1.0)$ \\
\hline
\end{tabular}

IQR: Interquartile range; ${ }^{\mathrm{a}}$ Other infections were one case of prostate abscess with $E$. coli, four cases of epididymitis, two cases of acute and one case of chronic prostatitis, and one case of pouchitis

(81.2\% of isolates). Most patients presented with UTI, with a minority of patients $(70,7.8 \%)$ presenting with an urosepsis. In nine patients we found other diagnoses that may explain a positive urine culture (Table 1). Of the 1,100 isolates $16(1.5 \%)$ were identified as E. coli producing extended spectrum betalactamase (ESBL).

\section{Uni-and Multivariate Analysis of Isolates From Patients Treated in 2007}

The multivariate analysis showed that increasing age, male gender, and a community acquirement of E. coli are independent risk factors for resistance to ciprofloxacin (Table 2). The rate of resistant isolates was not influenced by the clinical significance (i.e., colonization vs UTI) or by whether the urine was taken from a urinary catheter.

Isolates of uncomplicated UTI, which usually presented in women 40 years of age or younger $(n=48)$, showed resistance rates of $4.2 \%$ to ciprofloxacin, $20.8 \%$ to TMP/SMX, $10.4 \%$ to amoxicillin/clavulanate, and $8.3 \%$ to nitrofurantoin.

In 2007 , a total of 106/1,100 (9.9\%) E. coli urinary first isolates were sent by general practitioners to the micro- biology laboratory. Interestingly, the resistance rates were lower in these specimens compared to the overall data for ciprofloxacin (5.7\% vs 15.9\% overall), TMP/SMX (16.0\% vs $21.3 \%$ ), amoxicillin/clavulanate ( $5.7 \%$ vs $14.5 \%)$, and nitrofurantoin $(3.8 \%$ vs $5.3 \%)$. The difference was significant for ciprofloxacin (OR 0.29, 95\% CI 0.13-0.68, $\mathrm{p}=0.002$ ) and amoxicillin/clavulanate (OR $0.33,95 \% \mathrm{CI}$ $0.14-0.76, p=0.006)$ in the univariate analysis. However, after adjustment for age and sex, the difference was solely significant for amoxicillin/clavulanate (OR 0.34, 95\% CI $0.16-0.92, \mathrm{p}=0.03)$.

\section{Discussion}

Our study of 3,430 urinary first isolates of $E$. coli shows a trend of increasing resistance from 1997 to 2007, chiefly for ciprofloxacin, which parallels the increase in ciprofloxacin consumption in Switzerland. Our data indicate that the overall cumulative susceptibility data should be adjusted for age and gender if used as a basis for recommendations of the empirical antimicrobial therapy for UTIs.

\section{Secular Trends of Antimicrobial Resistance}

We observed an increase in ciprofloxacin resistance from $1.8 \%$ to $15.9 \%$ within 10 years, which is similar to that reported by other European countries [24] and the USA [15]. The increase in ciprofloxacin resistance correlates closely with increased ciprofloxacin consumption in Switzerland, as previously shown in the ECO-SENS study for other countries [25]. Although antibiotic use in Switzerland is generally low in comparison with other European nations, Switzerland is one of the leading nations in fluoroquinolone consumption (1.8 defined daily doses/day per 1,000 inhabitants) $[26,27]$, which may explain the rather high resistance rates for ciprofloxacin that we found in our study.

In contrast to fluoroquinolones, there was an inverse correlation between the number of TMP/SMX packages sold and resistance over time. As recently speculated for Moraxella catarrhalis and Haemophilus influenzae [28], the selection of TMP/SMX-resistant strains may be caused by co-resistance. This hypothesis was corroborated by the high rate $(51 \%)$ of co-resistance against TMP/SMX in ciprofloxacin-resistant isolates in our study.

Table 2

Uni- and multivariate analysis of risk factors for ciprofloxacin resistance in 1,100 urinary first isolates of $E$. coli in 2007.

\begin{tabular}{|c|c|c|c|c|}
\hline Variable & Crude OR (95\% CI) & p-value & Adjusted OR (95\% CI) & p-value \\
\hline Male gender & $1.47(1.02-2.22)$ & 0.04 & $1.59(1.05-2.41)$ & 0.03 \\
\hline Age (+10 years) & $1.32(1.19-1.46)$ & $<0.001$ & $1.29(1.15-1.45)$ & $<0.001$ \\
\hline Nosocomial isolate & $0.51(0.34-0.76)$ & $<0.001$ & $0.44(0.28-0.67)$ & $<0.001$ \\
\hline Urinary catheter & $1.15(0.76-1.75)$ & 0.50 & $1.21(0.76-1.91)$ & 0.42 \\
\hline Asymptomatic bacteriuria or colonization & $1.03(0.70-1.50)$ & 0.90 & $1.04(0.70-1.54)$ & 0.85 \\
\hline
\end{tabular}




\section{Characteristics of Patients with E. coli Urinary Isolates in 2007}

In line with results reported in earlier studies $[8,15]$, we found that ciprofloxacin resistance increased with the age of the patient, from $5.3 \%$ in those younger than 35 years of age to $21.9 \%$ in patients older than 75 years. In contrast, the resistance rate for TMP/SMX decreased with age. It is likely that older patients with UTIs were primarily treated with fluoroquinolones in recent years because the treating physicians falsely assumed that in the elderly TMP/SMX resistance is most frequent.

Our finding that ciprofloxacin resistance is more prevalent in males than in females confirms a recent report [15]. This may be explained by the different nature of UTIs in males with only complicated UTIs by definition resulting in fluoroquinolone therapy.

In contrast to other pathogens, fluoroquinolone resistance has not been reported to be higher in in-hospital isolates [7, 29], which may be explained by the widespread use of fluoroquinolones in the out-patient settings. Nosocomial E. coli UTIs are mainly due to factors associated with the hospital setting, such as the placement of urinary catheters during elective surgery. Although fluoroquinolone consumption is high in Switzerland, the consumption - and therefore the selective pressure - at our hospital is rather low (2008: 6.4 defined daily doses/100 patient days), which may explain the comparably low fluoroquinolone resistance in nosocomial isolates in our study. Similarly, E. coli isolates from urine specimens sent by general practitioners were less frequently resistant against ciprofloxacin and amoxicillin/ clavulanate. However, after adjustment for age and sex, this difference disappeared as patients with UTIs seen by general practitioners are generally younger and more frequently female. Taken together, cumulative antimicrobial susceptibility test data published by microbiology laboratories using hospital data is not necessarily accurate for the situation met by general practitioners.

Clinical information is lacking in most of the large epidemiological studies that have been published on the susceptibility rates of $E$. coli $[7,14,15,19]$. In contrast to other studies $[8,30]$, we did not find differences in the resistance pattern of $E$. coli isolates from patients presenting with a UTI compared to those with colonization.

In summary, the main limitation in the use of cumulative resistance data for establishing guidelines for empirical antibiotic treatment of UTIs is age. Based on our overall cumulative susceptibility test data, TMP/SMX should not be used empirically, as the resistance rate is higher than $20 \%$. However, our study does reveal that TMP/SMX may be used in patients in the over-75 age group (resistance rate $18 \%$ ). In contrast, ciprofloxacin can be safely used in patients younger than 55 years of age (resistance rate below 10\%), although the overall cumulative resistance rate was $19 \%$.
There are some limitations to our study. Due to its retrospective character, certain information, such as previous antibiotic treatment(s), could not be obtained. Only a small and possibly not sufficiently representative number of isolates originating from patients of general practitioners were available. A selection bias of patients presenting with more severe infections is likely because the samples were not prospectively taken. The definition of 'nosocomial' was limited by the lack of knowledge of hospitalization at other institutions before admission to our hospital. Furthermore, the time from application of a urinary catheter up to the time of urinary culture was unknown. Another limitation is that data on the amount of ciprofloxacin use were available only as the number of packages sold annually, but defined daily doses, as suggested by the World Health Organization, would probably be more accurate.

\section{Conclusion}

Resistance in urinary isolates of E. coli is emerging, chiefly for ciprofloxacin, and reflects the increased use of antimicrobial compounds. Cumulative resistance rates and surveillance networks may not predict E. coli resistance on an individual level because they are limited by the important differences in resistance rates with regard to age and gender. Therefore, guidelines for empirical therapy of UTI should be based on a knowledge of individualized resistance that takes - minimally - age and gender into consideration.

\section{Acknowledgments}

We thank IMS HEALTH (Hergiswil, Switzerland) for providing us with the data on antibiotic use in Switzerland and Marc Dangel for providing us with some of the data for this study.

Conflict of interest statement. A non-restricted grant from the "Stiftung Forschung Infektionskrankheiten, Basel", Switzerland. Ursula Flückiger and Manuel Battegay have received grants for the antibiotic stewardship program by: Astra Zeneca AG; Bristol-Myers Squibb SA; GlaxoSmithKline AG; Merck Sharp \& Dohme-Chibret AG; Wyeth Pharmaceuticals AG. The authors were independent of all funding and were free of conflicts of interest.

\section{References}

1. Stamm WE, Norrby SR: Urinary tract infections: disease panorama and challenges. J Infect Dis 2001; 183: S1-4.

2. Hillier S, Bell J, Heginbothom M, Roberts Z, Dunstan F, Howard A, Mason B, Butler CC: When do general practitioners request urine specimens for microbiology analysis? The applicability of antibiotic resistance surveillance based on routinely collected data. J Antimicrob Chemother 2006; 58: 1303-1306.

3. Colgan R, Johnson JR, Kuskowski M, Gupta K: A prospective study of risk factors for trimethoprim-sulfamethoxazole resis- 
tance in acute uncomplicated cystitis. Antimicrob Agents Chemother 2008; 167: 2201-2206.

4. Falagas ME, Polemis M, Alexiou VG, Marini-Mastrogiannaki A, Kremastinou J, Vatopoulos AC: Antimicrobial resistance of Esherichia coli urinary isolates from primary care patients in Greece. Med Sci Monit 2008; 14: CR75-CR79.

5. Hooton TM, Scholes D, Gupta K, Stapleton AE, Roberts PL, Stamm WE: Amoxicillin-clavulanate vs ciprofloxacin for the treatment of uncomplicated cystitis in women: a randomized trial. JAMA 2005; 293: 949-955.

6. Gupta K, Hooton TM, Stamm WE: Increasing antimicrobial resistance and the management of uncomplicated communityacquired urinary tract infections. Ann Intern Med 2001; 135: 41-50.

7. Karlowsky JA, Kelly $\sqcup$, Thornsberry C, Jones ME, Sahm DF: Trends in antimicrobial resistance among urinary tract infection isolates of Escherichia coli from female outpatients in the United States. Antimicrob Agents Chemother 2002; 46: 2540-2545.

8. Alos JI, Serrano MG, Gomez-Garces JL, Perianes J: Antibiotic resistance of Escherichia coli from community-acquired urinary tract infections in relation to demographic and clinical data. Clin Microbiol Infect 2005; 11: 199-203.

9. Huang ES, Stafford RS: National patterns in the treatment of urinary tract infections in women by ambulatory care physicians. Arch Intern Med 2002; 162: 41-47.

10. Warren JW, Abrutyn E, Hebel JR, Johnson JR, Schaeffer AJ, Stamm WE: Guidelines for antimicrobial treatment of uncomplicated acute bacterial cystitis and acute pyelonephritis in women. Infectious Diseases Society of America (IDSA). Clin Infect Dis 1999; 29: 745-758.

11. Taur Y, Smith MA: Adherence to the Infectious Diseases Society of America guidelines in the treatment of uncomplicated urinary tract infection. Clin Infect Dis 2007; 44: 769-774.

12. Stamm WE: Evaluating guidelines. Clin Infect Dis 2007; 44: $775-776$.

13. Gupta K, Hooton TM, Stamm WE: Isolation of fluoroquinoloneresistant rectal Escherichia coli after treatment of acute uncomplicated cystitis. J Antimicrob Chemother 2005; 56: $243-246$.

14. Gagliotti C, Nobilio L, Moro ML: Emergence of ciprofloxacin resistance in Escherichia coli isolates from outpatient urine samples. Clin Microbiol Infect 2007; 13: 328-331.

15. Becnel Boyd L, Atmar RL, Randall GL, Hamill RJ, Steffen D, Zechiedrich L: Increased fluoroquinolone resistance with time in Escherichia coli from $>17,000$ patients at a large county hospital as a function of time, culture site, age, sex, and location. BMC Infect Dis 2008; 8: 4 .

16. Arslan H, Azap OK, Ergonul O, Timurkaynak F: Risk factors for ciprofloxacin resistance among Escherichia coli strains isolated from community-acquired urinary tract infections in Turkey. J Antimicrob Chemother 2005; 56: 914-918.

17. Sannes MR, Belongia EA, Kieke B, Smith K, Kieke A, Vandermause $M$, Bender J, Clabots C, Winokur P, Johnson JR: Predictors of antimicrobial-resistant Escherichia coli in the feces of vegetarians and newly hospitalized adults in Minnesota and Wisconsin. J Infect Dis 2008; 197: 430-434.
18. Sandel DC, Wang CT, Kessler S: Urinary tract infections and a multidrug-resistant Escherichia coli clonal group. N Engl J Med 2002; 346: 535-536.

19. McDonald LC, Chen FJ, Lo HJ, Yin HC, Lu PL, Huang CH, Chen $P$, Lauderdale TL, Ho M: Emergence of reduced susceptibility and resistance to fluoroquinolones in Escherichia coli in Taiwan and contributions of distinct selective pressures. Antimicrob Agents Chemother 2001; 45: 3084-3091.

20. Brown PD, Freeman A, Foxman B: Prevalence and predictors of trimethoprim-sulfamethoxazole resistance among uropathogenic Escherichia coli isolates in Michigan. Clin Infect Dis 2002; 34: 1061-1066.

21. Blot S, Vandijck D, Vandewoude K, Depuydt P, Vogelaers D, Peleman R: Comment on: impact of antibiotic resistance and of adequate empirical antibiotic treatment in the prognosis of patients with Escherichia coli bacteraemia. J Antimicrob Chemother 2007; 60: 1402.

22. Peralta G, Sanchez MB, Garrido JC, De Benito I, Cano ME, Martinez-Martinez L, Roiz MP: Impact of antibiotic resistance and of adequate empirical antibiotic treatment in the prognosis of patients with Escherichia coli bacteraemia. J Antimicrob Chemother 2007; 60: 855-863.

23. Hindler JF, Evangelista AT, Jenkins SG, Johnston J, Master R, McGowan Jr. JE, Stelling J: Analysis and presentation of cumulative antimicrobial susceptibility test data; approved guideline (2nd edn). Clinical and Laboratory Standards Institute, Wayne, PA 2005

24. Nys S, Terporten PH, Hoogkamp-Korstanje JA, Stobberingh EE: Trends in antimicrobial susceptibility of Escherichia coli isolates from urology services in The Netherlands (1998-2005). J Antimicrob Chemother 2008; 62: 126-132.

25. Kahlmeter G: Prevalence and antimicrobial susceptibility of pathogens in uncomplicated cystitis in Europe. The ECO.SENS study. Int J Antimicrob Agents 2003; 22 (Suppl 2): 49-52.

26. Filippini M, Masiero G, Moschetti K: Socioeconomic determinants of regional differences in outpatient antibiotic consumption: evidence from Switzerland. Health Policy 2006; 78: 77-92.

27. Ferech M, Coenen S, Malhotra-Kumar S, Dvorakova K, Hendrickx $\mathrm{E}$, Suetens C, Goossens H: European surveillance of antimicrobial consumption (ESAC): outpatient quinolone use in Europe. J Antimicrob Chemother 2006; 58: 423-427.

28. Karpanoja P, Nyberg ST, Bergman M, Voipio T, Paakkari P, Huovinen $\mathrm{P}$, Sarkkinen $\mathrm{H}$ : Connection between trimethoprimsulfamethoxazole use and resistance in Streptococcus pneumoniae, Haemophilus influenzae, and Moraxella catarrhalis. Antimicrob Agents Chemother 2008; 52: 2480-2485.

29. Stelling JM, Travers K, Jones RN, Turner PJ, O'Brien TF, Levy SB: Integrating Escherichia coli antimicrobial susceptibility data from multiple surveillance programs. Emerg Infect Dis 2005; 11: 873-882.

30. Talan DA, Krishnadasan A, Abrahamian FM, Stamm WE, Moran GJ: Prevalence and risk factor analysis of trimethoprim-sulfamethoxazole- and fluoroquinolone-resistant Escherichia coli infection among emergency department patients with pyelonephritis. Clin Infect Dis 2008; 47: 1150-1158. 